\title{
Mortalidade por suicídio: perfil epidemiológico em um estado da Amazônia
}

\section{Brasileira}

\author{
Suicide mortality: epidemiological profile in a Brazilian Amazon state \\ Mortalidade por suicidio: perfil epidemiológico en un estado de la Amazonía brasileña
}

Fernanda Géssica da Silva Duarte

ORCID: https://orcid.org/0000-0002-1960-6446 Universidade Federal do Amapá, Brasil E-mail: fernandagessica15@gmail.com

Luana Jaçanã Resende dos Santos Tavares

ORCID: https://orcid.org/0000-0002-1031-464X Universidade Federal do Amapá, Brasil

E-mail: luanajacana@gmail.com

João Pedro Botelho de Mont'Alverne

ORCID: https://orcid.org/0000-0002-3947-8533 Universidade Federal do Amapá, Brasil

E-mail: j.pedromontalverne@ hotmail.com

Mailze Tainara Rodrigues Fonseca

ORCID: https://orcid.org/0000-0002-3393-7698 Universidade Federal do Amapá, Brasil E-mail: mailzer32@gmail.com

Maria Helena Mendonça de Araújo

ORCID: https://orcid.org/0000-0002-7742-144X Universidade Federal do Amapá, Brasil E-mail: ma.helenama@gmail.com Rosilene Ferreira Cardoso

ORCID: https://orcid.org/0000-0002-5834-8443 Universidade Federal do Amapá, Brasil E-mail: rosilenecardoso7@gmail.com

\begin{abstract}
Resumo
Objetivo: Analisar o perfil epidemiológico dos óbitos por suicídio no Estado do Amapá, no período de 2010 a 2019. Metodologia: Estudo epidemiológico retrospectivo transversal, de abordagem quantitativa e descritiva, através de dados secundários do Sistema de Informação de Mortalidade (SIM). Utilizaram-se informações referentes às mortes por lesões autoprovocadas intencionalmente (X60-X84, conforme CID-10), segundo o sexo e a faixa etária, que ocorreram de 2010 a 2019 no Estado do Amapá. Resultados: Foram registrados 425 óbitos por suicídio, entre 2010 e 2019, dos quais 339 foram do sexo masculino e 86 do sexo feminino (razão de 3,94:1). No mesmo período, constatouse aumento de $68,64 \%$ da taxa de mortalidade geral, sendo que nos homens, esse aumento foi de $62,86 \%$, e nas mulheres, de $92,65 \%$. Quanto à causa de suicídio e à faixa etária, respectivamente, observou-se que a maioria ocorreu por lesão autoprovocada de forma intencional causada por enforcamento, estrangulamento e sufocação (X70), e o maior percentual de mortes foi entre 20 e 29 anos (35,05\%), tanto no sexo masculino quanto no feminino. Conclusões: O suicídio é um problema complexo com grande impacto na saúde pública mundial e que vem apresentando crescimento nos últimos anos, como evidenciado neste estudo. Portanto, é salutar desenvolver análises epidemiológicas que auxiliem na identificação de grupos em risco de suicídio, a fim de compreender os aspectos envoltos nesse fenômeno, bem como seja possível adaptar intervenções para que haja maior eficácia e alcance na abordagem dessas populações.
\end{abstract}

Palavras-chave: Epidemiologia; Suicídio; Amazônia; Saúde Pública; Brasil.

\begin{abstract}
Objective: To analyze the epidemiological profile of the suicide deaths in the State of Amapá, in the period from 2010 to 2019. Methods: Retrospective transversal epidemiological study, with a quantitative and descriptive approach, through secondary data from the Mortality Information System (SIM). Information regarding deaths from intentionally self-inflicted injuries were used (X60-X84, according to CID-10), by sex and age group, that happened from 2010 to 2019 in the State of Amapá. Results: There were 425 deaths by suicide, between 2010 and 2019, of which 339 were male and 86 female (ratio of 3.94:1). In the same period, there was an increase of $68,64 \%$ in the general mortality rate, being that in men the increase was of $62.86 \%$, and in women, of $92.65 \%$. Concerning the cause of the suicide and the age group, respectively, it was observed that the majority occurred by intentionally self-
\end{abstract}


inflicted injury caused by hanging, strangulation and suffocation (X70), and the highest percentage of deaths was between 20 and 29 years old (35.05\%), both in male and females. Conclusions: Suicide is a complex problem with a great impact on public health worldwide and that has been growing in recent years, as evidenced in this study. Therefore, it is healthy to develop epidemiological analyzes that help to identify groups at risk of suicide, in order to understand the aspects involved in this phenomenon, as well as adapting interventions so that there is greater effectiveness and reach in addressing these populations.

Keywords: Epidemiology; Suicide; Amazonian Ecosystem; Public Health; Brazil.

\section{Resumen}

Objetivo: Analizar el perfil epidemiológico de las muertes por suicidio en el estado de Amapá, de 2010 a 2019. Metodología: Estudio epidemiológico transversal, retrospectivo, con enfoque cuantitativo y descriptivo. Se utilizaron datos secundarios del Sistema de Información de Mortalidad (SIM) sobre las muertes ocurridas entre 2010 y 2019 por autolesión intencional (X60-X84, CIE-10) por sexo y grupo de edad en el estado de Amapá. Resultados: Entre 2010 y 2019 se registraron 425 muertes por suicidio, de los cuales 339 eran hombres y 86 mujeres (relación 3,94:1). En el mismo período, hubo un aumento del 68,64\% en la tasa de mortalidad general, entre los hombres este aumento fue del $62,86 \%$ y entre las mujeres, del $92,65 \%$. En cuanto a la causa del suicidio y el grupo de edad, respectivamente, se observó que, entre hombres y mujeres, la mayoría ocurrió por autolesiones causadas intencionalmente por ahorcamiento, estrangulamiento y asfixia (X70), y el mayor porcentaje de muertes entre los adultos 20 y 29 años $(35,05 \%)$. Conclusiones: El suicidio es un problema complejo con un gran impacto en la salud pública a nivel mundial y como se evidencia en este estudio, ha ido creciendo en los últimos años en Amapá. Por ello, es importante desarrollar análisis epidemiológicos que ayuden a identificar grupos en riesgo de suicidio, con el fin de comprender los aspectos involucrados en este fenómeno y desarrollar intervenciones para que exista una mayor efectividad y cobertura el cuidado de estas poblaciones.

Palabras clave: Epidemiología; Suicidio; Ecosistema Amazónico; Salud Pública; Brasil.

\section{Introdução}

O suicídio é um problema complexo com grande impacto na saúde pública mundial, visto que além de representar uma tragédia individual, afeta indiretamente famílias, comunidades e a sociedade em geral, resultando em altos custos humanitários e econômicos (Cerel et al., 2019; DeBastiani \& De Santis, 2018; Turecki \& Brent, 2016).

O termo suicídio foi primeiramente usado no século XVII e deriva das palavras "sui", que significa a si mesmo, e “cidium”, que significa o ato de matar (DeBastiani \& De Santis, 2018). O conceito de suicídio, por sua vez, é a morte fruto de um ato executado pelo próprio indivíduo, de maneira a erradicar uma condição desesperadora de sofrimento, estando ele consciente do desfecho final (Pereira et al., 2019). Vários fenótipos são considerados no espectro do suicídio, sendo a nomenclatura historicamente heterogênea e não plenamente padronizada, destacando-se as denominações: suicídio, comportamento suicida, tentativa de suicídio, ideação suicida, autolesão, e autolesão não suicida (Turecki et al., 2019).

Nesse contexto, o termo comportamento suicida se refere aos comportamentos autoinfligidos, sejam fatais ou não, que podem resultar no fim da vida, revelando diferenças significativas com relação à influência genética, aos gêneros, às faixas etárias e às realidades sociopolíticas, que culminam em diferentes fatores de risco e heterogeneidade etiológica, já que componentes culturais, sociais, intelectuais, econômicos, psiquiátricos, psicológicos, filosófico-existenciais, isoladamente ou em conjunto, levam o indivíduo à prática do suicídio (Bahia et al., 2017; Turecki \& Brent, 2016; Turecki et al., 2019).

No cenário atual, destacam-se fatores de risco como: bullying e cyber-bullying (especialmente na infância e adolescência); maus-tratos na infância; história familiar de transtornos mentais, abuso ou de suicídio; transtornos alimentares; transtornos de humor (principalmente ansiedade e depressão) e de personalidade (com destaque à bipolaridade); abuso de álcool e drogas; não ter religião; solidão; perdas pessoais; conflitos na comunidade de convívio; trauma psicológico recente; insuficiência socioeconômica; baixa escolaridade; relacionamentos interpessoais conturbados; violência sexual; e ser portador de doença crônica e/ou incapacitante (Knipe et al., 2018; Ministério da Saúde, 2019; Raue, 2014; Ribeiro et al., 2018).

O suicídio é uma questão séria, que está dentro das principais causas de mortalidade no mundo, sendo responsável por cerca de 700.000 mortes anualmente, o que corresponde a um óbito a cada minuto. Dessa forma, a meta prioritária da Organização Mundial de Saúde (OMS) é reduzir a mortalidade por suicídio por meio de estratégias de prevenção maciça 
(World Health Organization [WHO], 2021).

De maneira geral, os suicídios são responsáveis por $1,4 \%$ das mortes prematuras em todo o mundo. Apesar de em cada país a realidade da situação ser diferente, nota-se que no que diz respeito: à idade, há maior incidência na segunda e terceira décadas de vida; ao sexo, o suicídio consumado é três vezes mais comum em homens do que em mulheres, embora estas apresentem maior número de tentativas; aos métodos utilizados, com maior frequência são enforcamento, envenenamento com pesticidas e uso de armas de fogo (Bachmann, 2018).

Ainda que haja dados e análises sobre o tema, acredita-se que os números de suicídio ainda são uma subestimação dos casos reais, tendo em vista que, às vezes, o ato suicida não é reconhecido ou relatado, por sua natureza sensível e ao tabu sobre a problemática; as mortes por suicídio podem ser classificadas incorretamente como um acidente ou outra causa de morte; e a ilegalidade do ato em certos contextos sociais, levando a um processo complicado para o registro de um suicídio, muitas vezes envolvendo autoridades judiciais (Bilsen, 2018; Silva et al., 2018).

Diante disso, os dados preliminares do Estado do Amapá indicam uma convergência com o aspecto nacional. Contudo, informações mais concretas e estudos mais detalhados são precários (Superintendência de Vigilância em Saúde do Amapá, 2020). Logo, o presente estudo se propôs a descrever o perfil epidemiológico dos óbitos por suicídio no Estado do Amapá considerando as mortes por lesões autoprovocadas intencionalmente (X60-X84), conforme a décima edição da Classificação Estatística Internacional de Doenças e Problemas relacionados com a Saúde (CID 10), durante o período de 2010 a 2019, encontradas na pesquisa conforme a causa da morte segundo o sexo e a faixa etária.

\section{Metodologia}

Trata-se de um estudo epidemiológico retrospectivo transversal, de abordagem quantitativa e natureza descritiva (Pereira et al., 2018). Utilizaram-se os bancos de dados retiradas do Sistema de Informação de Mortalidade (SIM) para óbitos causados por lesões autoprovocadas intencionalmente (X60-X84, conforme CID-10), que ocorreram no período de 2010 a 2019 no Estado do Amapá.

Os critérios de inclusão foram: os óbitos por suicídio ocorridos no Estado do Amapá no período de 2010 a 2019, classificados dentro do CID X60 a X84 e disponíveis no SIM. Os casos que não foram notificados ou com causa de morte indefinida ou ignorada ou cujos dados não estavam disponíveis no SIM não entraram na análise desta pesquisa.

As causas de morte foram agrupadas pelos especificadores em autointoxicação por exposição intencional (X60 a X69) e lesão autoprovocada intencionalmente (X70 a X84). Para as variáveis sociodemográficas utilizou-se o sexo (masculino e feminino) e a idade agrupada por faixa etária (5 a 14 anos, 15 a 19 anos, 20 a 39 anos, 40 a 59 anos, 60 a 79 anos, 80 anos ou mais e idade ignorada).

Depois de coletados, os dados foram armazenados em planilhas no Microsoft Office Excel versão 2016 para a tabulação e posterior elaboração de gráficos e tabelas. Após tabulação, foram calculadas as frequências absolutas e relativas encontradas para cada variável; a taxa de mortalidade anual; a mortalidade proporcional por sexo e faixa etária e a tendência em relação à taxa de mortalidade e ao ano.

As estimativas populacionais do Amapá foram retiradas do site do Instituto Brasileiro de Geografia e Estatística (IBGE, 2020) e selecionadas segundo o sexo (masculino e feminino) e a faixa etária (5 a 14 anos, 15 a 19 anos, 20 a 29 anos, 30 a 39 anos, 40 a 49 anos, 50 a 59 anos, 60 a 69 anos, 70 a 79 anos, 80 anos ou mais).

A taxa de mortalidade anual foi calculada segundo o sexo conforme a faixa etária a partir dos dados do SIM e IBGE para cada causa específica conforme a fórmula (Rede Interagencial de Informações para a Saúde, [RIPSA], 2020): 


$$
\text { Taxa de mortalidade }=\frac{\begin{array}{c}
N \text { de ábitos pela causa específica, } \\
\text { em determinado local e período }
\end{array}}{\begin{array}{c}
\text { População total do mesmo } \\
\text { local e período }
\end{array}} \times 100.000
$$

Para o cálculo da mortalidade proporcional segundo o sexo e a faixa etária utilizaram-se os dados do SIM para cada causa específica conforme a fórmula (Fundação Oswaldo Cruz, 2017):

$$
\text { Mortalidade proporcional }=\frac{\begin{array}{c}
N o \text { de óbitos pela causa específica } \\
\text { segundo faixa etárias } \\
\text { em determinado local e período }
\end{array}}{\begin{array}{c}
\text { Notal de óbitos pela cousa específica } \\
\text { no mesmo local e período }
\end{array}}
$$

Esta pesquisa utilizou dados secundários de sistemas de informação e notificação. Assim, este estudo, segundo a resolução nº 510/2016 do Conselho Nacional de Saúde, dispensa encaminhamento e aprovação do Comitê de Ética e Pesquisa.

\section{Resultados}

Entre 2010 e 2019 foram registrados 425 óbitos por suicídio no Estado do Amapá. Destes, 339 foram de indivíduos do sexo masculino e 86 do sexo feminino, levando a uma razão de cerca de 3,94 óbitos masculinos para cada óbito feminino. O ano com a maior razão foi 2013, com 8 óbitos em homens para cada óbito em mulheres. Já o ano com a menor razão foi 2018, com 2,1 óbitos masculinos para cada óbito feminino. Em 2019, a razão ficou em 3,36 óbitos no sexo masculino para cada óbito no sexo feminino.

Embora no Sistema de Informação de Mortalidade (SIM) existam 21 categorias para causa de morte por suicídio, divididas em autointoxicação por exposição intencional (X60 a X69) e lesão autoprovocada intencionalmente (X70 a X84) foram identificadas apenas 12 categorias para os sexos masculino e feminino nessa população.

Os óbitos masculinos notificados abrangeram as causas de autointoxicação por exposição intencional ao/às: drogas anticonvulsivantes (antiepilépticos), sedativos, hipnóticos, antiparkinsonianos e psicotrópicos não classificados em outra parte (X61) e pesticidas (X68), com 2 mortes cada, ou seja, 0,59\% das mortes; outras drogas, medicamentos e substâncias biológicas não especificadas (X64); álcool (X65) e outros produtos químicos e substâncias nocivas não especificadas (X69) foi registrada 1 morte em cada categoria, $0,29 \%$. Além disso, foram registrados óbitos no grupo das lesões autoprovocadas de forma intencional causadas por: enforcamento, estrangulamento e sufocação (X70) 307 mortes, 90,56\%, o maior número entre as categorias; disparo de arma de fogo de mão (X72) 10 mortes, 2,95\%; disparo de outra arma de fogo e de arma de fogo não especificada (X74) 6 mortes, 1,77\%; afogamento e submersão (X71), objeto contundente (X79) e precipitação de um lugar elevado (X80) com 3 mortes cada, $0,88 \%$.

Já nos óbitos em mulheres, além das causas já citadas de X65 (1,16\%) e X68 (1,16\%) cada uma com 1 morte, X69 $(3,49 \%)$ com 3 mortes e X70 (93,02\%) com 80 mortes, identificou-se nessa população 1 morte por lesão autoprovocada intencionalmente por meios não especificados (X84), contabilizando 1,16\%.

A taxa de mortalidade foi calculada pela fórmula: ( $\mathrm{N}^{\circ}$ de óbitos por suicídio no Estado do Amapá, nos anos de 2010 e $2019 \div$ População do Estado do Amapá, nos anos de 2010 e 2019) x 100.000. A Tabela 1 mostra as taxas de mortalidade por causa de suicídio, no ano de 2010 e 2019, em homens, mulheres e no geral para os dois sexos. Nota-se que a taxa de mortalidade geral (X64-X84) sofreu um aumento de 68,64\% quando se compara 2010 com 2019. Nos homens, esse aumento foi de $62,86 \%$, e nas mulheres, de $92,65 \%$. Em relação a cada causa de suicídio, observa-se que X70 foi a principal tanto no sexo masculino quanto no feminino e nos dois anos detalhados, representando $86,72 \%$ da taxa de mortalidade em 2010 , e $98,37 \%$ da taxa em 2019. Nos homens, essa causa correspondeu a 87,42\% e 97,87\% em 2010 e 2019, respectivamente. E nas mulheres, $83,05 \%$ e $100 \%$, respectivamente. 
Research, Society and Development, v. 11, n. 1, e48711125151, 2022

(CC BY 4.0) | ISSN 2525-3409 | DOI: http://dx.doi.org/10.33448/rsd-v11i1.25151

Tabela 1 - Taxas de mortalidade conforme a causa de morte e o sexo, anos 2010 e 2019.

\begin{tabular}{c|c|c|c|c|c|c}
\hline \multirow{2}{*}{$\begin{array}{c}\text { Causa de } \\
\text { Morte }\end{array}$} & \multicolumn{3}{|c|}{$\mathbf{2 0 1 0}$} & \multicolumn{2}{c}{$\mathbf{2 0 1 9}$} \\
\cline { 2 - 7 } & Homens & Mulheres & Geral & Homens & Mulheres & Geral \\
\hline X61 & 0,00 & - & 0,00 & 0,00 & - & 0,00 \\
\hline X64 & 0,00 & - & 0,00 & 0,24 & - & 0,12 \\
\hline X65 & 0,00 & 0,00 & 0,00 & 0,00 & 0,00 & 0,00 \\
\hline X68 & 0,00 & 0,00 & 0,00 & 0,00 & 0,00 & 0,00 \\
\hline X69 & 0,00 & 0,29 & 0,15 & 0,00 & 0,00 & 0,00 \\
\hline X70 & 6,05 & 1,47 & 3,79 & 11,03 & 3,41 & 7,25 \\
\hline X71 & 0,00 & - & 0,00 & 0,00 & - & 0,00 \\
\hline X72 & 0,58 & - & 0,29 & 0,00 & - & 0,00 \\
\hline X74 & 0,29 & - & 0,15 & 0,00 & - & 0,00 \\
\hline X79 & 0,00 & - & 0,00 & 0,00 & - & 0,00 \\
\hline X80 & 0,00 & - & 0,00 & 0,00 & - & 0,00 \\
\hline Total & - & 0,00 & 0,00 & - & 0,00 & 0,00 \\
\hline
\end{tabular}

Fonte: Autores segundo SIM (Tabnet-DataSus) e IBGE conforme dados disponíveis em junho de 2020.

O Gráfico 1 demostra a curva de tendência de regressão da taxa de mortalidade padronizada por suicídio/100.000 habitantes em ambos os sexos, de acordo com o gênero no período estudado. O crescimento da taxa de mortalidade não ocorreu de maneira linear diferente do esperado a partir da linha de tendência gerada. Durante o período houve uma flutuação dos valores das taxas de mortalidade demonstrada pelo valor $\mathrm{R}^{2}$ baixo das três curvas. As taxas de mortalidade masculina e geral foram as que mais se aproximaram da linha de tendência a partir do ano de 2017. Já na feminina ocorreu o contrário, com as taxas de mortalidade mais próximas ao modelo nos primeiros cinco anos do estudo. Nos homens, tem-se a menor taxa sendo relatada em 2012 (4,41) e a maior em 2019 (11,27). Já nas mulheres, a menor taxa foi em 2014 (1,08) e a maior em 2018 $(4,96)$. Na população geral, a menor taxa foi em $2012(2,92)$ e a maior em $2018(7,63)$.

Gráfico 1 - Curva de tendência de regressão das taxas de mortalidade por suicídio conforme o gênero, 2010 a 2019.

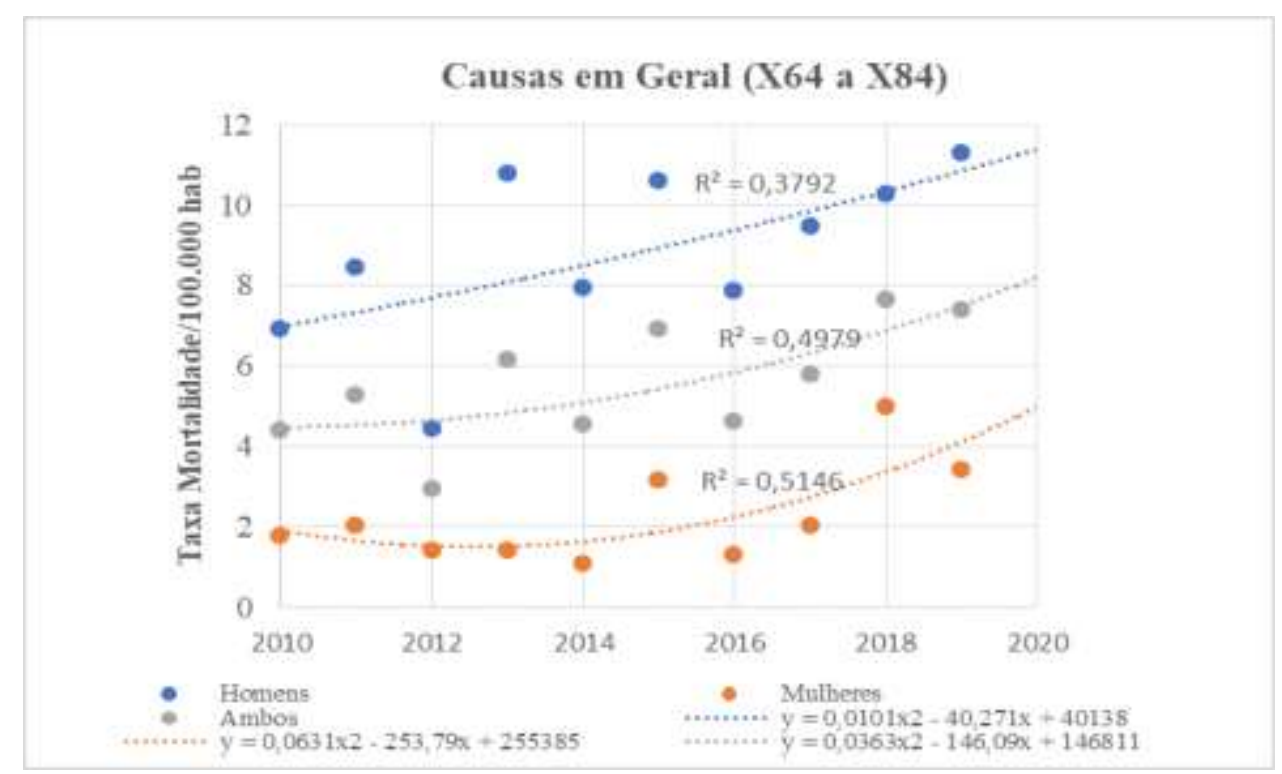

Fonte: Autores segundo SIM (Tabnet-DataSus) e IBGE conforme dados disponíveis em junho de 2020.

O Gráfico 2 expõe a tendência da taxa de mortalidade em cada sexo, no período estudado, da principal causa de óbito, isto é, X70. Embora a tendência seja de crescimento nas taxas de mortalidade por X70 na população em geral e para cada sexo, a taxa de mortalidade masculina sofreu bastante flutuação no período. É possível identificar os anos de 2012 e 2013 como os 
mais distantes estatisticamente da linha de tendência gerada. Além disso, o valor baixo de $\mathrm{R}^{2}$ na curva dos homens também demonstra essa flutuação. Na taxa geral, tem-se o ponto mais baixo sendo em 2012 (2,78) e o mais alto em 2019 (7,25). Nos homens, os anos com menor e maior taxa coincidiram com os dados encontrados na população em geral, em $2012(4,13)$ e 2019 (11,03), respectivamente. Já nas mulheres, o ano com a menor taxa foi $2016(1,03)$ e o com a maior taxa foi $2019(4,21)$.

Gráfico 2 - Curva de tendência de regressão das taxas de mortalidade (CID X70) conforme o gênero, 2010 a 2019.

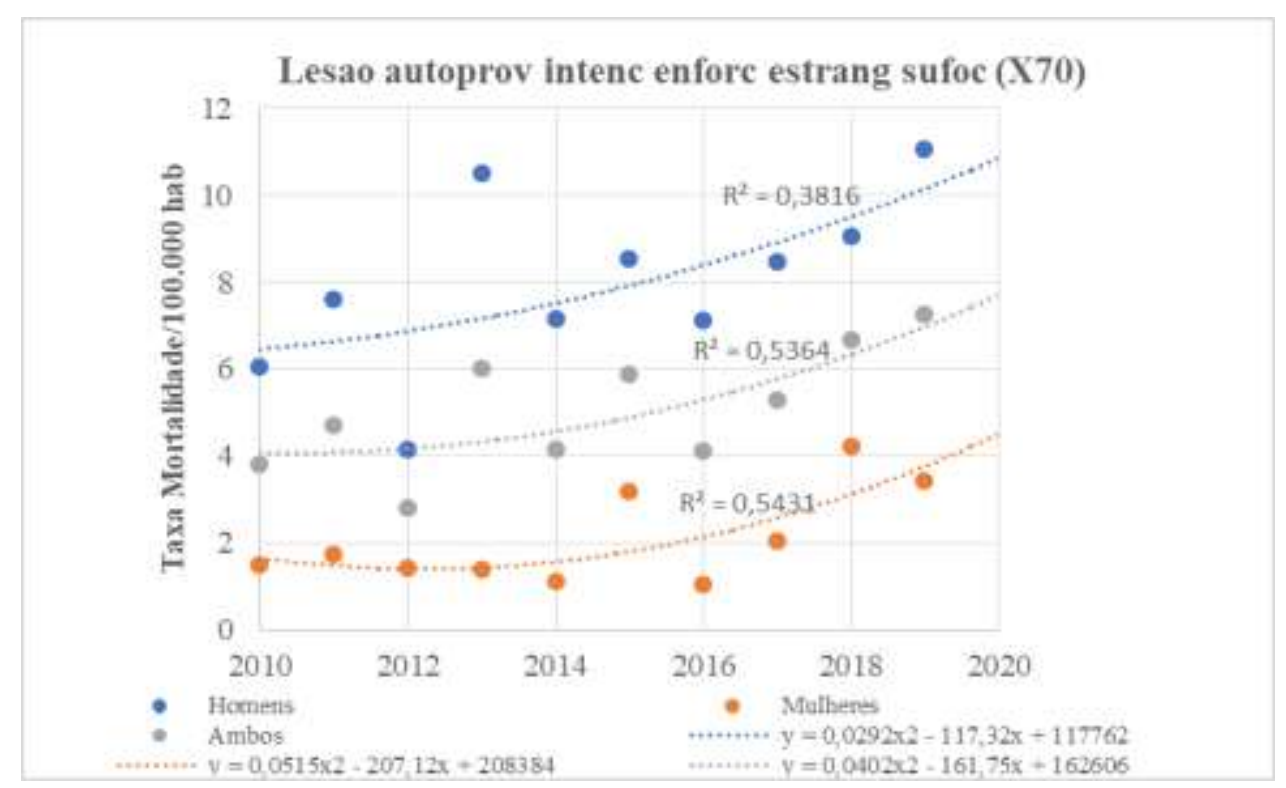

Fonte: Autores segundo SIM (Tabnet-DataSus) e IBGE conforme dados disponíveis em junho de 2020.

Em relação aos óbitos por faixa etária (anos), a TABELA 02 apresenta o número de óbitos em homens e mulheres, por faixa etária. Analisando-se os números totais somados de todas as categorias (X61 a X84), tem-se que o percentual de mortes, do maior para o menor, foi entre: 20 e 29 anos (35,05\%), 30 e 39 anos (19,05\%), 15 e 19 anos (17,17\%), 40 e 49 anos (10,11\%), 50 e 59 anos (7,52\%), 60 e 69 anos (3,29\%), 70 e 79 anos (2,82\%), 5 e 9 anos (0,47\%) e acima de 80 anos $(0,23 \%)$. Nos dois sexos e em quase todas as faixas etárias, os óbitos por Lesão Autoprovocada Intencionalmente Por Enforcamento, Estrangulamento e Sufocação foram os mais substanciais, correspondendo a 91,1\% de todos os óbitos. As faixas etárias com as maiores taxas de mortalidade proporcional por X70 foram: idosos de 60 a 69 anos (100\%), jovens de 15 a 19 anos (95,9\%) e adultos de 20 a 29 anos (93,3\%). Ademais, a única faixa etária sem óbitos por X70 foi a de idosos acima de 80 anos, sendo identificado apenas 1 óbito por X80 durante todo o período estudado. 
Research, Society and Development, v. 11, n. 1, e48711125151, 2022

(CC BY 4.0) | ISSN 2525-3409 | DOI: http://dx.doi.org/10.33448/rsd-v11i1.25151

Tabela 2 - Óbitos por suicídio segundo a faixa etária e o sexo, 2010 a 2019.

\begin{tabular}{|c|c|c|c|c|c|c|c|c|c|c|c|}
\hline \multirow[b]{2}{*}{$\begin{array}{l}\text { Causa da } \\
\text { morte }\end{array}$} & \multicolumn{11}{|c|}{ Faixa etária (em anos) } \\
\hline & & 5 a 9 & $\begin{array}{c}10 \mathrm{a} \\
14\end{array}$ & $\begin{array}{c}15 \text { a } \\
19\end{array}$ & $\begin{array}{c}20 \text { a } \\
29\end{array}$ & $\begin{array}{c}30 \text { a } \\
39\end{array}$ & $\begin{array}{c}40 \mathrm{a} \\
49\end{array}$ & $\begin{array}{c}50 \text { a } \\
59\end{array}$ & $\begin{array}{c}60 \mathrm{a} \\
69\end{array}$ & $\begin{array}{c}70 \mathrm{a} \\
79\end{array}$ & $\begin{array}{c}> \\
80\end{array}$ \\
\hline \multirow{2}{*}{ X61 } & Homens & 0 & 0 & 0 & 1 & 0 & 0 & 1 & 0 & 0 & 0 \\
\hline & Mulheres & 0 & 0 & 0 & 0 & 0 & 0 & 0 & 0 & 0 & 0 \\
\hline \multirow{2}{*}{ X64 } & Homens & 0 & 0 & 0 & 0 & 1 & 0 & 0 & 0 & 0 & 0 \\
\hline & Mulheres & 0 & 0 & 0 & 0 & 0 & 0 & 0 & 0 & 0 & 0 \\
\hline \multirow{2}{*}{ X65 } & Homens & 0 & 0 & 0 & 1 & 0 & 0 & 0 & 0 & 0 & 0 \\
\hline & Mulheres & 0 & 0 & 0 & 0 & 0 & 0 & 1 & 0 & 0 & 0 \\
\hline \multirow{2}{*}{ X68 } & Homens & 0 & 0 & 0 & 0 & 1 & 1 & 0 & 0 & 0 & 0 \\
\hline & Mulheres & 0 & 0 & 0 & 1 & 0 & 0 & 0 & 0 & 0 & 0 \\
\hline \multirow{2}{*}{ X69 } & Homens & 0 & 0 & 0 & 1 & 0 & 0 & 0 & 0 & 0 & 0 \\
\hline & Mulheres & 0 & 1 & 2 & 0 & 0 & 0 & 0 & 0 & 0 & 0 \\
\hline \multirow{2}{*}{$\mathrm{X} 70$} & Homens & 0 & 10 & 44 & 117 & 61 & 30 & 24 & 12 & 9 & 0 \\
\hline & Mulheres & 1 & 6 & 26 & 22 & 9 & 10 & 3 & 2 & 1 & 0 \\
\hline \multirow{2}{*}{ X71 } & Homens & 1 & 0 & 0 & 1 & 1 & 0 & 0 & 0 & 0 & 0 \\
\hline & Mulheres & 0 & 0 & 0 & 0 & 0 & 0 & 0 & 0 & 0 & 0 \\
\hline \multirow{2}{*}{$\mathrm{X} 72$} & Homens & 0 & 0 & 1 & 0 & 5 & 1 & 2 & 0 & 1 & 0 \\
\hline & Mulheres & 0 & 0 & 0 & 0 & 0 & 0 & 0 & 0 & 0 & 0 \\
\hline \multirow{2}{*}{$X 74$} & Homens & 0 & 0 & 0 & 2 & 2 & 1 & 1 & 0 & 0 & 0 \\
\hline & Mulheres & 0 & 0 & 0 & 0 & 0 & 0 & 0 & 0 & 0 & 0 \\
\hline \multirow{2}{*}{$\mathrm{X} 79$} & Homens & 0 & 0 & 0 & 2 & 1 & 0 & 0 & 0 & 0 & 0 \\
\hline & Mulheres & 0 & 0 & 0 & 0 & 0 & 0 & 0 & 0 & 0 & 0 \\
\hline \multirow{2}{*}{$\mathrm{X} 80$} & Homens & 0 & 0 & 0 & 1 & 0 & 0 & 0 & 0 & 1 & 1 \\
\hline & Mulheres & 0 & 0 & 0 & 0 & 0 & 0 & 0 & 0 & 0 & 0 \\
\hline \multirow{2}{*}{ X84 } & Homens & 0 & 0 & 0 & 0 & 0 & 0 & 0 & 0 & 0 & 0 \\
\hline & Mulheres & 0 & 1 & 0 & 0 & 0 & 0 & 0 & 0 & 0 & 0 \\
\hline \multicolumn{2}{|c|}{ Total } & 2 & 18 & 73 & 149 & 81 & 43 & 32 & 14 & 12 & 1 \\
\hline
\end{tabular}

Fonte: Autores segundo SIM (Tabnet-DataSus) conforme dados disponíveis em junho de 2020.

A Tabela 3 evidencia a taxa de mortalidade por X70 na população em geral segundo a faixa etária no período de 2010 a 2019. Com relação às maiores taxas de mortalidade, nota-se que em 2010 concentrava-se em pessoas com 70 a 79 anos (23,54); em 2011 e 2012 foi entre pessoas de 60 a 69 anos (10,21 e 9,58, respectivamente); em 2013, a taxa voltou a ser superior nos indivíduos entre 70 e 79 anos (30,05). Entretanto, nos anos de 2014 e 2015, a maior taxa foi observada entre jovens de 15 e 19 anos (12,47 e 12,17, respectivamente). Em 2016, 2017 e 2018, as maiores taxas de mortalidade foram identificadas entre os indivíduos com, respectivamente: 50 a 59 anos (9,32), 60 e 69 anos (13,72), 70 a 79 anos (15,43). Já em 2019, a maior taxa de mortalidade foi observada em indivíduos com 20 a 29 anos $(15,32)$.

Tabela 3 - Taxas de mortalidade por X70 na população em geral segundo a faixa etária, 2010 a 2019.

\begin{tabular}{c|c|c|c|c|c|c|c|c|c|c}
\hline $\begin{array}{c}\text { Faixa etária } \\
\text { (em anos) }\end{array}$ & $\mathbf{2 0 1 0}$ & $\mathbf{2 0 1 1}$ & $\mathbf{2 0 1 2}$ & $\mathbf{2 0 1 3}$ & $\mathbf{2 0 1 4}$ & $\mathbf{2 0 1 5}$ & $\mathbf{2 0 1 6}$ & $\mathbf{2 0 1 7}$ & $\mathbf{2 0 1 8}$ & $\mathbf{2 0 1 9}$ \\
\hline $\mathbf{5}$ a 9 & 0,00 & 1,16 & 0,00 & 0,00 & 0,00 & 0,00 & 0,00 & 0,00 & 0,00 & 0,00 \\
\hline $\mathbf{1 0}$ a 14 & 1,26 & 1,22 & 0,00 & 1,17 & 1,15 & 3,41 & 0,00 & 0,00 & 5,77 & 4,65 \\
\hline $\mathbf{1 5}$ a 19 & 6,93 & 6,75 & 1,31 & 11,51 & 12,47 & 12,17 & 3,54 & 6,90 & 12,41 & 11,12 \\
\hline $\mathbf{2 0}$ a 29 & 6,97 & 7,61 & 6,73 & 12,49 & 6,49 & 9,89 & 6,21 & 11,44 & 13,77 & 15,32 \\
\hline $\mathbf{3 0}$ a 39 & 3,98 & 7,70 & 4,66 & 5,41 & 7,00 & 9,36 & 5,80 & 4,85 & 6,33 & 5,42 \\
\hline $\mathbf{4 0}$ a 49 & 1,50 & 4,30 & 1,37 & 6,53 & 3,75 & 4,80 & 6,92 & 5,56 & 4,30 & 8,30 \\
\hline $\mathbf{5 0}$ a 59 & 7,70 & 7,26 & 4,58 & 6,51 & 0,00 & 1,96 & 9,32 & 5,33 & 5,09 & 6,48 \\
\hline $\mathbf{6 0}$ a 69 & 5,42 & 10,21 & 9,58 & 0,00 & 0,00 & 7,86 & 7,34 & 13,72 & 0,00 & 6,01 \\
\hline $\mathbf{7 0}$ a 79 & 23,54 & 0,00 & 0,00 & 30,05 & 0,00 & 0,00 & 0,00 & 8,13 & 15,43 & 7,29 \\
\hline$\geq 80$ & 0,00 & 0,00 & 0,00 & 0,00 & 0,00 & 0,00 & 0,00 & 0,00 & 0,00 & 0,00 \\
\hline
\end{tabular}

Fonte: Autores segundo SIM (Tabnet-DataSus) e IBGE conforme dados disponíveis em junho de 2020.

Por fim, as faixas etárias com as maiores taxas de mortalidade por X70 na população masculina foram observadas nos idosos de 70 a 79 anos de idade nos anos de $2013(63,3)$ e 2010 (49,98); e em adultos de 20 a 29 anos em 2019 (23,99). Nas 
mulheres, as três maiores taxas de mortalidade para a mesma causa de morte foram observadas no ano de 2018 entre jovens de 15 a 19 anos (15,75) e idosas de 70 a 79 anos (14,83); e em 2011 em adultas de 30 a 39 anos (15,38).

\section{Discussão}

O suicídio constitui importante causa de mortalidade na atualidade a nível global, representando cerca de 1,3\% das mortes da população geral em 2019 (WHO, 2021). Conforme estudos de séries temporais no Brasil, o Norte é uma das regiões com as menores taxas de óbitos por suicídio no país (Mata et al., 2020; Santos Júnior et al., 2019). Apesar disso, o presente estudo identificou aumento no número de mortes por lesões autoprovocadas de forma intencional no Estado do Amapá durante o período de 2010 a 2019, com tendência de crescimento como visto no Gráfico 01, concordando com os padrões nacionais deste evento (Leal et al., 2020; Mata et al., 2020).

Em 2019, na população geral em estudo, foram registradas 7,37 mortes para cada 100.000 habitantes, como demonstrado na Tabela 01, números que ultrapassam os dados nacionais mais recentes divulgados pela Organização Mundial de Saúde (WHO, 2021), os quais apontam taxa de mortalidade por suicídio de 6,4 a cada 100.000 habitantes no Brasil para o mesmo ano.

O número de óbitos foi maior de forma estatisticamente significativa em indivíduos do sexo masculino $(79,76 \%)$ para a população estudada, em relação ao sexo feminino (20,24\%), atingindo uma razão de 3,94:1. Tais dados são similares com estudos e padrões nacionais em que a taxa proporcional de suicídios por homens foi 3,4 vezes maior do que a das mulheres (Lee et al., 2019; Tavares et al., 2020; WHO, 2021).

Quanto aos métodos empregados no ato do suicídio, a partir da análise da série temporal, observa-se que a maior proporção de mortes ocorreu devido às lesões causadas por enforcamento, estrangulamento e sufocação entre a população masculina (90,56\%), assim como na população feminina (93,02\%). Para os homens, outros meios mais comumente empregados também estavam dentro das causas por lesão autoprovocada intencionalmente (7,37\%), enquanto que para as mulheres foi prevalente a autointoxicação por exposição intencional $(5,81 \%)$, concordando com a literatura nacional e mundial (Ministério da Saúde, 2019; Pedrosa et al., 2018; Rosa et al., 2016).

Vale ressaltar que estudos apontam para um cenário no qual as mulheres apresentam maior risco de tentativa de suicídio enquanto os homens o maior risco de morte, fato este que envolve os métodos de lesão autoprovocada, tendo em vista que a letalidade do método escolhido é um forte fator de risco para a conclusão do ato. Geralmente são empregados meios com maior potencial de letalidade no sexo masculino, enquanto as mulheres se apropriam de métodos de menor invasão letal (Ministério da Saúde, 2017; Ministério da Saúde, 2019; Miranda-Mendizabal et al., 2019).

De acordo com a Tabela 02, no que se refere à faixa etária, a maior prevalência de óbitos por suicídio foi notificada em indivíduos com idade entre 20 e 29 anos (35,06\%), com padrão crescente nos últimos anos, seguido dos indivíduos entre 30 e 39 anos (19,06\%). Tal resultado também foi encontrado em outros estudos epidemiológicos nacionais (Beriguel et al., 2020; Pedrosa et al., 2018). Santos Júnior e colaboradores (2019) similarmente encontraram maiores taxas em indivíduos jovens adultos entre 15-29 anos (50,1\%) e 30-49 anos (32,3\%) no estado de Alagoas.

Além do mais, a população adolescente e jovem entre 15 a 19 anos contribuiu com parcela significativa dos casos de óbitos por suicídio (17,18\%), acompanhando tendência global, de acordo com dados da OMS (WHO, 2021). Sabe-se que a transição entre infância e vida adulta é rodeada de fatores estressantes como mudanças corporais, hormonais, comportamentais e psicológicas, cobrança com estudos e mercado de trabalho, conflitos familiares, introdução a substâncias lícitas e ilícitas, entre outros, que podem culminar com um estado de maior vulnerabilidade psicológica, aumentando os riscos de ideações e comportamentos suicidas nesse público (Carballo et al., 2020; Wanzinack et al., 2017). 
Ao analisar a variação da taxa de mortalidade pela principal causa de óbito no estudo (X70) na Tabela 03, entre os anos de 2010 a 2019, pode-se constatar crescimento significativo na população masculina de 15 a 19 anos (117,98\%) e queda significativa para a mesma faixa etária na população feminina (-21,28\%). Nos idosos ocorreu queda entre os homens de 70 a 79 anos (-69,61\%), e nas mulheres não foi possível a mensuração, uma vez que em 2010 e 2019 não foram encontrados registros para essa faixa etária.

Considerando a população idosa, fatores micro e macrossociais como viuvez, perdas de amigos próximos, isolamento social, redução do desempenho físico, declínio cognitivo, dependência para atividades diárias, uso de múltiplos fármacos e maior frequência de doenças com potencial de sofrimento físico ou psíquico, são relatados como relevantes para a prática suicida nessa população (Conejero et al., 2018; Santos et al., 2019). Crestani et al. (2019) encontraram como principais métodos empregados para suicídio nesta população o enforcamento, precipitação de local elevado, afogamento, disparo por arma de fogo e envenenamento, o que se assemelha ao detectado no presente estudo, em que do total de 27 óbitos em pessoas acima de 60 anos, 88,9\% foram por enforcamento, estrangulamento e sufocação (X70), 7,4\% por precipitação de um lugar elevado (X80) e 3,7\% resultante de disparo de arma de fogo de mão (X72).

Por fim, é relevante ressaltar a ausência de estatísticas fidedignas sobre o suicídio de indivíduos LGBTQIA+ (lésbicas, gays, bissexuais, transexuais, travestis, transgêneros, queer, intersexuais, assexuais e mais), o que pode ser justificado pela inexistência de notificação específica nos atestados de óbitos (Pedrosa et al., 2018). Fatores como discriminação social, não aceitação por familiares ou indivíduos próximos, rejeição, agressão física e psicológica são alguns das intempéries que envolvem a classe, tornando-os uma população exposta e suscetível a comprometimento da saúde mental, distúrbios psiquiátricos (principalmente os de humor) e comportamentos autolesivos, com destaque a ideações suicidas, enquadrando a população LGBTQIA+ como possível exposta ao maior risco de suicídio nas mais variadas idades, incluindo a adolescência (Cerel et al., 2020; Guz et al., 2021; Mark et al., 2020).

\section{Conclusão}

A partir da análise dos resultados, identificou-se aumento significativo nas taxas de mortalidade entre a população de 10 a 29 anos no Estado do Amapá, bem como a maioria das mortes no sexo masculino e a causa morte mais prevalente por enforcamento, estrangulamento e sufocação (X70). Espera-se que este trabalho de cunho epidemiológico contribua para a identificação de grupos específicos em risco de suicídio de modo que intervenções possam ser adaptadas para uma maior eficácia e alcance na abordagem dessas populações. Mais pesquisas devem ser realizadas para identificar os perfis psicológicos ou eventos traumáticos que tornem os sujeitos nestas faixas etárias mais vulneráveis. Ademais, apesar dos números expostos nesta pesquisa, deve-se lembrar da ocorrência de subnotificação dos casos de violência autoprovocada de forma intencional que resultam em morte, devido a fatores como o estigma associado ao suicídio e dificuldade da identificação do evento.

Diante deste cenário, almeja-se que as secretarias de vigilância epidemiológica e da saúde, nas esferas estadual e municipal, reforcem a importância da correta notificação compulsória da causa morte. Considera-se, ainda, que o tema suicídio deve ser abordado no processo de formação e capacitação de profissionais das áreas da saúde, educação e demais prestadores de serviço envolvidos no atendimento das populações mais vulneráveis, visando identificar e acolher esses pacientes através de assistência psicológica especializada, reduzindo o sofrimento dos mesmos e as taxas de mortalidade no Estado. Por fim, a realização de estudos futuros com análises qualitativas sobre os demais aspectos envoltos no comportamento suicida, tais quais sofrimento psíquico, crenças e discriminação; assim como o levantamento do perfil epidemiológico dos casos nas regiões brasileiras podem contribuir para uma melhor perspectiva dessa temática. 


\section{Agradecimentos}

Os autores agradecem à Michele Maleamá Sfair, do departamento de Suicídio e Lesões Autoprovocadas da Superintendência de Vigilância em Saúde do Amapá, por nos receber na superintendência, explicar como é o funcionamento do sistema adotado na instituição e compartilhar os boletins epidemiológicos do Estado do Amapá.

\section{Referências}

Bachmann, S. (2018). Epidemiology of Suicide and the Psychiatric Perspective. International Journal of Environmental Research and Public Health, 15 (7), 1425. https://doi.org/10.3390/ijerph15071425.

Bahia, C. A., Avanci, J. Q., Pinto, L. W., \& Minayo, M. C. de S. (2017). Lesão autoprovocada em todos os ciclos da vida: perfil das vítimas em serviços de urgência e emergência de capitais do Brasil. Ciência \& Saúde Coletiva, 22 (9), 2841-2850. https://doi.org/10.1590/1413-81232017229.12242017.

Beringuel, B. M., Costa, H. V. V., Silva, A. P. S. C., \& Bonfim, C. V. (2020). Mortalidade por suicídio no Estado de Pernambuco, Brasil (1996-2015). Revista Brasileira de Enfermagem, 73 (Suppl 1), e20180270. https://doi.org/10.1590/0034-7167-2018-0270.

Bilsen, J. (2018). Suicide and Youth: Risk Factors. Frontiers in Psychiatry, 9, 540. https://doi.org/10.3389/fpsyt.2018.00540.

Carballo, J. J., Llorente, C., Kehrmann, L., Flamarique, I., Zuddas, A., Purper-Ouakil, D., Hoekstra, P. J., Coghill, D., Schulze, U., Dittmann, R. W., Buitelaar, J. K., Castro-Fornieles, J., Lievesley, K., Santosh, P., Arango, C., \& STOP Consortium (2020). Psychosocial risk factors for suicidality in children and adolescents. European Child \& Adolescent Psychiatry, 29 (6), 759-776. https://doi.org/10.1007/s00787-018-01270-9.

Cerel, J., Brown, M. M., Maple, M., Singleton, M., van de Venne, J., Moore, M., \& Flaherty, C. (2019). How Many People Are Exposed to Suicide? Not Six. Suicide and Life-Threatening Behavior, 49 (2), 529-534. https://doi.org/10.1111/s1tb.12450.

Cerel, J., Tucker, R. R., Aboussouan, A., \& Snow, A. (2021). Suicide exposure in transgender and gender diverse adults. Journal of Affective Disorders, 278, 165-171. https://doi.org/10.1016/j.jad.2020.09.045.

Conejero, I., Olié, E., Courtet, P., \& Calati, R. (2018). Suicide in older adults: current perspectives. Clinical interventions in aging, 13, 691-699. https://doi.org/10.2147/CIA.S130670.

Crestani, C., Masotti, V., Corradi, N., Schirripa, M. L., \& Cecchi, R. (2019). Suicide in the elderly: a 37-years retrospective study. Acta bio-medica: Atenei Parmensis, 90 (1), 68-76. https://doi.org/10.23750/abm.v90i1.6312.

DeBastiani, S., \& De Santis, J. P. (2018). Suicide Lethality: A Concept Analysis. Issues in Mental Health Nursing, 39 (2), 117-125. https://doi.org/10.1080/01612840.2017.1364812.

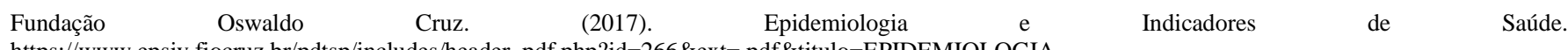
https://www.epsjv.fiocruz.br/pdtsp/includes/header_pdf.php?id=266\&ext=.pdf\&titulo=EPIDEMIOLOGIA.

Guz, S., Kattari, S. K., Atteberry-Ash, B., Klemmer, C. L., Call, J., \& Kattari, L. (2021). Depression and Suicide Risk at the Cross-Section of Sexual Orientation and Gender Identity for Youth. The Journal of adolescent health: official publication of the Society for Adolescent Medicine, 68 (2), 317-323. https://doi.org/10.1016/j.jadohealth.2020.06.008.

Instituto Brasileiro de Geografia e Estatística. (2020). Cidades e Estados. https://cidades.ibge.gov.br/brasil/ap/panorama.

Knipe, D. W., Gunnell, D., Pearson, M., Jayamanne, S., Pieris, R., Priyadarshana, C., Weerasinghe, M., Hawton, K., Konradsen, F., Eddleston, M., \& Metcalfe, C. (2018). Attempted suicide in Sri Lanka - An epidemiological study of household and community factors. Journal of affective disorders, 232, 177184. https://doi.org/10.1016/j.jad.2018.01.028.

Leal, C. B., Silva, I. S., \& Veloso, L. C. (2020). Epidemiological profile and incidence of a suicide victims in Brazil. Research, Society and Development, 9 (10), e7989109256. https://doi.org/10.33448/rsd-v9i10.9256.

Lee, S., Dwyer, J., Paul, E., Clarke, D., Treleaven, S., \& Roseby, R. (2019). Differences by age and sex in adolescent suicide. Australian and New Zealand Journal of Public Health, 43 (3), 248-253. https://doi.org/10.1111/1753-6405.12877.

Mak, J., Shires, D. A., Zhang, Q., Prieto, L. R., Ahmedani, B. K., Kattari, L., Becerra-Culqui, T. A., Bradlyn, A., Flanders, W. D., Getahun, D., Giammattei, S. V., Hunkeler, E. M., Lash, T. L., Nash, R., Quinn, V. P., Robinson, B., Roblin, D., Silverberg, M. J., Slovis, J., Tangpricha, V., ... Goodman, M. (2020). Suicide Attempts Among a Cohort of Transgender and Gender Diverse People. American Journal of Preventive Medicine, 59 (4), 570-577. https://doi.org/10.1016/j.amepre.2020.03.026.

Mata, K. C. R. da, Daltro, M. R., \& Ponde, M. P. (2020). Perfil epidemiológico de mortalidade por suicídio no Brasil entre 2006 e 2015. Revista Psicologia, Diversidade E Saúde, 9 (1), 74-87. https://doi.org/10.17267/2317-3394rpds.v9i1.2842.

Ministério da saúde. (2017). Boletim epidemiológico. Suicídio: Saber, agir e prevenir. 48 (30), 1-15. https://portalarquivos2.saude.gov.br/images/pdf/2017/setembro/21/2017-025-Perfil-epidemiologico-das-tentativas-e-obitos-por-suicidio-no-Brasil-e-a-rede-deatencao-a-saude.pdf.

Ministério da Saúde. (2019). Boletim epidemiológico. Suicídio: tentativas e óbitos por intoxicação exógena no Brasil, 2007 a 2016.50 (15), 1-12. https://antigo.saude.gov.br/images/pdf/2019/julho/17/2019-014-Publicacao-02-07.pdf.

Ministério da Saúde. (2020). Sistema de Informações sobre Mortalidade. http://www2.datasus.gov.br/DATASUS/index.php?area=060701. 
Miranda-Mendizabal, A., Castellví, P., Parés-Badell, O., Alayo, I., Almenara, J., Alonso, I., Blasco, M. J., Cebrià, A., Gabilondo, A., Gili, M., Lagares, C., Piqueras, J. A., Rodríguez-Jiménez, T., Rodríguez-Marín, J., Roca, M., Soto-Sanz, V., Vilagut, G., \& Alonso, J. (2019). Gender differences in suicidal behavior in adolescents and young adults: systematic review and meta-analysis of longitudinal studies. International journal of public health, 64 (2), 265-283. https://doi.org/10.1007/s00038-018-1196-1.

Pedrosa, N. F. C., Barreira, D. A., Rocha, D. Q. C., \& Barreira, M. A. (2018). Análise dos principais fatores epidemiológicos relacionados ao suicídio em uma cidade no interior do Ceará, Brasil. Journal of Health and Biological Sciences, 6 (4), 399-404. doi:10.12662/2317-3076jhbs.v6i4.2068.p399-404.2018.

Pereira, A. S., Shitsuka, D. M., Parreira, F. J., \& Shitsuka, R. (2018). Método Qualitativo, Quantitativo ou Quali-Quanti. In: Metodologia da Pesquisa Científica. https://repositorio.ufsm.br/bitstream/handle/1/15824/Lic_Computacao_Metodologia-Pesquisa-Cientifica.pdf?sequence=1.

Pereira, R. G., Conceição, B. B. da, Damasceno, Y. L. S., Oliveira, G. R. C., Cardoso, R. de M., Medeiros, J. M. de, Silva, N. A. da, Tinoco, K. F., Oliveira, V. dos S. da S. de, Salazar, A. A. da C., Santos, D. V. da S., Figueiredo, N. C. G. de A., Castro, K. R. de O., Viana, C. L. A., \& Fernandes, E. C. S. (2019) Análise do perfil do paciente suicida: revisão integrativa. Revista Eletrônica Acervo Saúde, (25), e607. https://doi.org/10.25248/reas.e607.2019.

Raue, P. J., Ghesquiere, A. R., \& Bruce, M. L. (2014). Suicide risk in primary care: identification and management in older adults. Current psychiatry reports, 16 (9), 466. https://doi.org/10.1007/s11920-014-0466-8.

Rede Interagencial de Informações para a Saúde. (2020). Taxa de mortalidade específica por causas externas (coeficiente de mortalidade específica por causas externas). Brasília - DF.

Ribeiro, J. D., Huang, X., Fox, K. R., \& Franklin, J. C. (2018). Depression and hopelessness as risk factors for suicide ideation, attempts and death: metaanalysis of longitudinal studies. The British journal of psychiatry: the journal of mental science, 212 (5), 279-286. https://doi.org/10.1192/bjp.2018.27.

Rosa, N. M., DellAgnolo, C. M., Oliveira, R. R., Mathias, T. A. F., Oliveira, \& M. L. F. (2016). Tentativas de suicídio e suicídios na atenção pré-hospitalar. Jornal Brasileiro de Psiquiatria, 65 (3), 231-238. https://doi.org/10.1590/0047-2085000000129.

Santos Júnior, C. J. dos, Santos, I. V., Silva, J. V. dos S., Gomes, V. de M., \& Ribeiro, M. C. (2019). Perfil de pacientes atendidos por tentativa de suicídio em um Hospital Geral de Emergências do estado de Alagoas, Brasil. Medicina (Ribeirão Preto), 52 (3), 223-230. https://doi.org/10.11606/issn.21767262.v52i3p223-230.

Santos, E. D. G. M., Rodrigues, G. O. L., Santos, L. M., Alves, M. E. S., Araújo, L. F., \& Santos, J. V. O. (2019). Suicídio entre idosos no Brasil: uma revisão de literatura dos últimos 10 anos. Psicología, Conocimiento y Sociedad, 9 (1), 205-220. https://dx.doi.org/10.26864/pcs.v9.n1.12.

Silva, B. F. A., Prates, A. A. P., Cardoso, A. A., \& Rosas, N. (2018). O suicídio no Brasil contemporâneo. Sociedade e Estado, 33 (2), $565-579$. https://doi.org/10.1590/s0102-699220183302014.

Superintendência de Vigilância em Saúde do Amapá. (2020). Boletim Epidemiológico: Situação Epidemiológica sobre Notificação de Lesão Autoprovocada e Óbitos por Suicídio no Amapá. Informe nº 02. https://editor.amapa.gov.br/arquivos_portais/publicacoes/SVS_fd37fda2d26838720ef9c8cdef089f75.pdf.

Tavares, F. L., Borgo, V. M. P., Leite, F. M. C., Cupertino, E. G. F., Pereira, J. A., Alves, R. N. R., \& Rosa, M. (2020). Mortalidade por suicídio no Espírito Santo, Brasil: uma análise do período de 2012 a 2016. Avances en Enfermería, 38 (1), 66-76. https://doi.org/10.15446/av.enferm.v38n1.79960.

Turecki, G., \& Brent, D. A. (2016). Suicide and suicidal behaviour. Lancet, 387 (10024), 1227-1239. https://doi.org/10.1016/S0140-6736(15)00234-2.

Turecki, G., Brent, D. A., Gunnell, D., O'Connor, R. C., Oquendo, M. A., Pirkis, J., \& Stanley, B. H. (2019). Suicide and suicide risk. Nature reviews. Disease primers, 5 (1), 74. https://doi.org/10.1038/s41572-019-0121-0.

Wanzinack, C., Temoteo, A., \& Oliveira, A. L. (2017). Mortalidade por suicídio entre adolescentes/jovens brasileiros: Um estudo com dados secundários entre os anos de 2011 a 2015. Revista Eletrônica Interdisciplinar, Matinhos, 10 (2), 106-117. http://dx.doi.org/10.5380/diver.v10i2.54974.

World Health Organization. (2021). Suicide worldwide in 2019: Global Health Estimates. https://www.who.int/publications/i/item/9789240026643. 